\title{
ORIENTACIONES MOTIVACIONALES, RENDIMIENTO ACADÉMICO Y GÉNERO EN ESTUDIANTES DE BACHILLERATO
}

\section{GOAL ORIENTATIONS, ACADEMIC ACHIEVEMENT AND GENDER IN COLLEGE STUDENTS}

\author{
María José Alemán \\ Universidad Católica del Uruguay, Uruguay \\ Daniel Trías \\ Universidad Católica del Uruguay, Uruguay \\ Karina Curione \\ Universidad de la República, Uruguay
}

\begin{abstract}
Resumen: Se presenta un estudio con estudiantes de bachillerato cuyo objetivo es indagar en qué medida la orientación motivacional y el género se reflejan en su rendimiento académico. Se trabajó con una muestra intencional de 116 alumnos, de un nivel socioeconómico medio-alto, que cursaban quinto año. Las orientaciones motivacionales (aprendizaje, evitación, lucimiento) fueron evaluadas mediante el cuestionario de auto-informe MEVA (Alonso Tapia, 2005). El rendimiento académico fue medido por el promedio de calificaciones en un conjunto de asignaturas. Se ha observado que el rendimiento académico difiere significativamente al considerar las distintas orientaciones motivacionales. Es la orientación al aprendizaje la que redunda en niveles más altos de rendimiento. Las mujeres obtienen niveles de rendimiento significativamente más altos. Se discute el papel de los contextos educativos que promueven diversidad contemplando distintas orientaciones motivacionales y considerando el géner
\end{abstract}

Palabras clave: orientación a metas, rendimiento académico, género, aprendizaje, psicología educacional.

\begin{abstract}
A study with college students is presented; this study aims to investigate to what extent the goal orientation and gender predict their academic achievement. The sample was a purposive sample of 116 students of medium-high social class of fifth degree. The motivational orientations (learning, avoidance and performance) were measured by the Self-report Questionnaire MEVA (Alonso Tapia, 2005). The academic achievement was measured by the average scores in a group of courses. The results show that the academic achievement is significantly different when the motivational orientations are considered. Orientation to learning led to the highest levels of achievement, as compared to avoidance and performance. Women scored higher in academic achievement. The role of educational contexts that promote the diversity are discussed, contemplating different motivational orientations and gender.
\end{abstract}

Keywords: goal orientations, academic achievement, gender, learning, educational psychology.

\section{INTRODUCCIÓN}

Se presentan los resultados de un estudio con estudiantes de bachillerato, a quienes se ha evaluado en sus orientaciones motivacionales y comparado en su rendimiento académico. Se busca indagar en qué medida la orientación motivacional de los sujetos y el género se reflejan en su rendimiento académico.

Difícilmente los actuales estudios sobre aprendizaje académico puedan dejar al margen los aspectos motivacionales (Rodríguez
Moneo y Huertas, 2000; Winne y Nesbit, 2010). Si bien se ha destacado el papel de la motivación en el aprendizaje académico como un factor modulador a la hora de enfrentar las tareas académicas (Alonso Tapia, 2005), la evidencia empírica disponible aún no es concluyente sobre el modo en el qué las variables motivacionales influyen en el rendimiento académico (Linnenbrink, 2005). Por este motivo resultan relevantes estudios que aporten evidencia sobre las relaciones entre motivación y rendimiento académico en contextos educativos. 
En este estudio se pretenden retomar preguntas vigentes para la investigación y práctica cotidiana en los ámbitos educativos. Centralmente se trata de conocer qué motiva a los estudiantes y cuáles son las consecuencias de su orientación motivacional sobre el rendimiento académico. Esta es una de las variables críticas para su éxito y permanencia en el sistema educativo (Pintrich, 2003).

\section{DE LAS METAS Y ORIENTACIONES MOTIVACIONALES}

Una característica de la conducta motivada el dirigirse hacia objetivos o propósitos. Por tanto, las metas han sido un componente motivacional que se ha estudiado especialmente en la psicología científica, generando variados sistemas de clasificación (Huertas, 2008). Particularmente, la teoría de orientación a metas constituye uno de los cuerpos de investigación más importantes a la hora de abordar el conocimiento de la motivación (Kaplan y Maehr, 2007).

Las metas que poseen los estudiantes organizan y regulan su comportamiento de cara a la consecución de un logro. Dichos logros se encuentran determinados por el tipo de orientación motivacional que adopten. Entre las múltiples metas posibles, el aprendizaje es uno de los objetivos que en contextos educativos se valora especialmente, aunque no siempre es lo que los alumnos jerarquizan.

Uno de los intentos más relevantes de resumir las variadas metas que las personas pueden perseguir, es la clasificación propuesta por Dweck y Elliot (1983). Estos autores describen dos patrones motivacionales básicos que pueden presentar los estudiantes en el contexto académico. Dichos patrones se definen en función de la meta que los alumnos se proponen alcanzar: metas de aprendizaje (learning goals) y metas de ejecución o rendimiento (performance goals). En primera instancia, se asume que la adopción de este último tipo de metas tiene efectos negativos en el rendimiento académico de los estudiantes (Dweck y Legget, 1988).

A posteriori se ha establecido una importante distinción entre dos tipos de metas de ejecución: metas de aproximación a la ejecución y metas de evitación de la ejecución (Elliot y Church,
1997; Elliot y Harackiewicz, 1996; Harackiewicz, Bower y Burg, 1998). Los estudiantes que se proponen metas centradas en la evaluación de su competencia desarrollan un patrón de motivación por la ejecución, que se centra en demostrar habilidad o competencia. A su vez podrían mostrar sus dos variantes motivacionales: aproximación, definida por el deseo de obtener una buena evaluación o una buena imagen (motivación por el lucimiento); evitación, caracterizada por el deseo de evitar evaluaciones negativas o evitar quedar mal (motivación por el miedo al fracaso).

La investigación en teoría de metas sugiere que la orientación a metas de aprendizaje conduce a niveles más altos de implicación cognitiva, afecto positivo e interés, más esfuerzo y persistencia en las tareas, búsqueda de ayuda y asunción de riesgos (Elliot y Dweck, 1988; Harackiewicz et al., 2000; Harackiewicz et al., 2002).

Por el contrario, la orientación a metas de ejecución conduce -en su variante de evitacióna mayores niveles de ansiedad, menor valor de la tarea, menor implicación cognitiva, disminución del esfuerzo, dejar de persistir en la tarea, así como niveles más bajos de rendimiento (Linnenbrink, 2005). Sin embargo, no resultan tan claras las implicaciones de la variante de lucimiento (aproximación) dado que los estudiantes que presentan este tipo de orientación obtienen tanto resultados positivos como negativos (Kaplan y Maehr, 2007).

Ahora bien, el concepto de orientación motivacional implica a las metas y abarca la experiencia de la persona. Se entiende que la orientación motivacional guía la interpretación de los conocimientos y la producción de cogniciones, emociones y comportamientos frente a las tareas (Elliott y Dweck, 1988). Estás orientaciones motivacionales se desarrollan y son altamente dependientes del contexto, aunque más investigación se requiere para dar cuenta del modo en qué los factores ambientales afectan la trayectoria de dichas orientaciones (Delgado y cols, 2010; Kaplan y Flum, 2010).

Como se ha señalado parte de la investigación sobre motivación se ha centrado en el estudio de las metas y su vinculación con los modos 
de autorregular los pensamientos, sentimientos y conductas en un contexto dado. Boekaerts y Corno (2005), distinguen dos grandes grupos de metas (de dominio y de bienestar) que los estudiantes persiguen y que se vinculan con los modos de regular pensamientos, sentimientos $y$ acciones en contextos educativos. En su modelo plantean una forma de procesamiento descendente (Top-down) en la que el sujeto parte de sus metas, determinando sus acciones para alcanzar los objetivos en un contexto dado. Sostienen que sujetos con metas de aprendizaje, tenderán a acciones de procesamiento profundo, que redundarán en su aprendizaje. Al mismo tiempo plantean una forma de procesamiento ascendente (Bottom-up), en que las claves del contexto activan sobretodo metas de bienestar cuando éste se vuelve amenazante.

En un esfuerzo similar, Effklides (2011) propone un modelo en el que busca describir la interacción entre las variables personales y las características de las tareas en un contexto dado. En este complejo modelo se hace visible que las orientaciones motivacionales pueden considerarse variables personales que afectan la autorregulación del aprendizaje. Esta investigadora propone como uno de los desafíos actuales identificar configuraciones de procesos personales que inciden en las conductas y en sus resultados. Las orientaciones motivacionales y el género pueden ser algunos de estos procesos.

\section{DE LA RELACIÓN ENTRE ORIENTACIONES MOTIVACIONALES Y RENDIMIENTO ACADÉMICO}

Por rendimiento académico se entiende un resultado que surge de un complejo proceso en el cual inciden múltiples variables (del sujeto, del contexto socio-educativo, del docente, etc.). El listado de variables que inciden en el rendimiento académico puede ser amplísimo y cambiar a medida que avanzan los estudiantes en el sistema educativo.

Risso, Peralbo y Barca (2010) identificaron 68 variables que van desde los estudios de los padres, la relación con pares, aspectos motivacionales, etc., y observaron cambios en distintas etapas de la enseñanza secundaria. En dicho estudio mostraban un rendimiento académico más homogéneo los estudiantes del grupo más avanzado en el sistema educativo.

Al abordar el rendimiento académico desde una perspectiva psicológica, Winne y Nesbit (2010) incluyen factores cognitivos, metacognitivos, motivacionales, contextuales que redundan en unas formas de afrontamiento de las tareas y unos resultados. En su esquema teórico que evidenciado la complejidad del rendimiento académico, que a su vez puede abordarse desde otras perspectivas.

Resulta claro que el rendimiento académico no puede considerarse un reflejo directo de las variables motivacionales. Sin embargo, analizar estas relaciones resulta relevante dado que resultan centrales para determinar las trayectorias educativas. A efectos del presente estudio se toma como medida del rendimiento académico el promedio de calificaciones en un conjunto de asignaturas, siendo ésta una aproximación fundamentalmente operativa (Rodríguez, 2010).

Motivación y rendimiento académico se encuentran íntimamente relacionados de forma bidireccional. Alonso Tapia (2001) señala que muchas veces los alumnos no aprenden porque no están motivados. En cambio, otras veces no están motivados porque no aprenden. En estos casos su modo de afrontar el aprendizaje y las estrategias utilizadas no son efectivas, esto les impide experimentar la sensación de competencia. Siendo éste uno de los factores centrales que motivan al estudiante.

Al analizar la relación entre las metas y el rendimiento académico, se observa que la adopción de metas de aprendizaje se ha relacionado positivamente con el uso de estrategias cognitivas y de autorregulación (Archer, 1994; Greene \& Miller, 1996; Harackiewicz et. al, 2000; Meece et. al, 1988; Valle et al, 2009), que redundarían en niveles más altos de rendimiento académico.

Por el contrario, se ha observado que las metas de evitación mantienen relaciones negativas con el uso de estrategias de procesamiento profundo, mientras se relacionan positivamente con el uso de estrategias de procesamiento superficial (Meece y Miller, 1990; Archer, 1994; Liem et al, 2008 en Valle et al, 2009). 
Como se ha señalado en relación a las metas de lucimiento, los estudios encontrados no son categóricos en sus implicaciones (Meece y Miller, 1990). En algunos trabajos se observan las consecuencias negativas de adoptar metas de lucimiento sobre el rendimiento. Mientras en otros se presentan resultados positivos, mostrando que las metas de lucimiento también pueden conducir al uso de estrategias de procesamiento más profundo y de autorregulación cognitiva (Harackiewicz et al., 2002). Se interpreta que centrarse en un criterio externo como "ser el mejor de la clase" conduciría a los estudiantes a implicarse más en las tareas académicas y en el uso de las estrategias cognitivas que les permiten alcanzar la meta propuesta (Pintrich y Schunk, 2006).

\section{ORIENTACIONES MOTIVACIONALES Y GÉNERO}

La investigación sobre motivación en contextos educativos ha mostrado que los estudiantes tienden a diferenciarse en algunos constructos motivacionales según el género. Se ha observado que los varones tienden a presentar mayor sensación de competencia que las mujeres, mientas ellas tienden a presentar niveles más altos de orientación a metas de aprendizaje (Midgley, Kaplan, \& Middlenton, 2001; Yeung, Lau \& Nie, 2011).

Cerezco y Casanova (2004) estudiaron diferencias de género en distintas variables cognitivo-motivacionales y el rendimiento en dos asignaturas (lenguaje y matemáticas). A partir de su estudio con 521 alumnos de educación secundaria, observaron la existencia de diferencias de género en las atribuciones causales, metas académicas y estrategias de aprendizaje significativo. Las mujeres presentan menores niveles de motivación extrínseca, se responsabilizan más de sus fracasos, utilizan en mayor medida estrategias de procesamiento de la información y obtuvieron mejor calificación en la asignatura de lenguaje que sus pares varones. Por otro lado, no observaron diferencias de género en las medidas de: autoconcepto académico, motivación intrínseca, atribuciones causales ante los éxitos y rendimiento académico en matemáticas.

De todos modos, debe señalarse, que no abundan los estudios que examinan las diferencias de género en el marco de la teoría de orientación a metas (Delgado y cols., 2010; Meece, Bower y Burg, 2006). A su vez, dichos estudios no muestran un patrón claro de diferencias de género en la orientación al logro de los estudiantes.

\section{En suma}

Constituye el problema de investigación determinar si las diferentes orientaciones motivacionales y el género se traducen en distintos niveles de rendimiento académico. El objetivo central de la presente investigación será analizar las diferencias de rendimiento académico de un grupo de estudiantes de bachillerato considerando sus orientaciones motivacionales y el género.

Volviendo a lo propuesto por Boekaerts y Corno (2005) en su modelo, se hipotetiza que sujetos con metas de aprendizaje, orientarán sus acciones al aprendizaje y, consecuentemente, obtendrán un rendimiento académico superior. En la misma línea se define como hipótesis que los sujetos con metas de bienestar (evitación y lucimiento), tratarán de poner a salvo su autoestima, no necesariamente encaminarán su acción al aprendizaje y consecuentemente alcanzarán niveles más bajos de rendimiento académico. También se analizarán diferencias de género expresadas en el rendimiento académico.

\section{MÉTODO}

\subsection{Participantes}

Se trata de una muestra intencional constituida por 116 alumnos, que cursaban quinto año de educación secundaria pertenecientes a un colegio privado de un nivel sociocultural medioalto. Un $42,7 \%$ de la muestra eran varones y un $57,2 \%$ eran mujeres.

\subsection{Instrumento}

El Cuestionario de motivación, expectativas y valor relacionado con el aprendizaje (MEVA, Alonso Tapia 2005): evalúa tres grandes orientaciones motivacionales (hacia el aprendizaje, resultado y la evitación) de los alumnos de se- 
cundaria y bachillerato. Los participantes deben responder señalando su grado de acuerdo, en una escala Lickert que varía de uno (totalmente en desacuerdo) a cinco (totalmente de acuerdo). Se ha utilizado la versión abreviada que consta de 75 elementos. Su validez predictiva es R: .496 (Alonso Tapia 2005).

\subsection{Diseño}

Se trata de un estudio "ex post facto". Las variables seleccionadas son: orientaciones motivacionales (aprendizaje, resultado y evitación), género, y rendimiento académico. La medida de rendimiento académico surge de considerar el promedio total entre la nota final y las notas correspondientes a las materias compartidas por todas las opciones académicas. Este promedio demostró ser una medida que correlaciona de forma positiva y significativa con las materias en común (Alemán, 2011).

\subsection{Procedimiento}

Se solicitó autorización al equipo directivo del colegio, para trabajar con los alumnos de quinto año de secundaria. Al inicio de la administración colectiva se brindó información a los estudiantes sobre el objetivo de la aplicación, la confidencialidad de los datos, así como también la elección de participar. Se entregó a cada estudiante el Cuestionario MEVA y una hoja de respuestas. Se registraron las calificaciones de cada materia y la nota final del período julioagosto de cada sujeto.

\section{RESULTADOS}

El objetivo que orienta la presentación de los resultados es analizar las diferencias de rendimiento académico de un grupo de estudiantes de bachillerato considerando sus orientaciones motivacionales y el género.

De la muestra más amplia (116 participantes) hemos considerado sólo aquellos sujetos que han obtenido puntuaciones mayores al percentil 70 en una ( $y$ solo en una) de tres las orientaciones motivacionales. De este modo se buscaron maximizar las diferencias entre los tres grupos constituidos por las distintas orientaciones motivacionales.
En la Tabla 1 pueden observarse las medias y las desviaciones estándar de rendimiento académico considerando las orientaciones motivacionales a la evitación, al aprendizaje y al lucimiento, y también el género.

Tabla 1.

Medias y desviaciones de rendimiento académico ordenadas por orientación motivacional y género.

\begin{tabular}{|cc|c|c|c|}
\hline & $\begin{array}{c}\text { O. Evitación } \\
n=20\end{array}$ & $\begin{array}{c}\text { O. Aprendizaje } \\
n=34\end{array}$ & $\begin{array}{c}\text { O. Lucimiento } \\
n=27\end{array}$ \\
\hline $\begin{array}{c}\text { Mujeres } \\
n=52\end{array}$ & Media & 5,97 & 7,04 & 6,71 \\
\hline Varones & Media & 5,34 & 7,16 & 1,10 \\
$n=29$ & $D S$ & 0,90 & 0,83 & 5,50 \\
\end{tabular}

Se utilizó un ANOVA factorial, dado que se busca analizar la incidencia de las orientaciones motivacionales y el género en el rendimiento académico de los estudiantes. Los estadísticos pueden observarse en la tabla 2.

Tabla 2.

Resumen de estadísticos del ANOVA factorial.

\begin{tabular}{|c|c|c|c|c|}
\hline & $\boldsymbol{F}$ & $\mathbf{g l}$ & $\begin{array}{c}\text { Eta al cuadrado } \\
\text { parcial }\end{array}$ & $\boldsymbol{p}$ \\
\hline $\begin{array}{c}\text { Orientación } \\
\text { motivacional }\end{array}$ & 8,24 & 2,75 &, 180 & .001 \\
\hline Género & 5,23 & 1,75 &, 065 & .025 \\
\hline $\begin{array}{c}\text { Orientación } \\
\text { motivacional } \\
\text { x Género }\end{array}$ & 1,66 & 2,75 &, 042 & .197 \\
\hline
\end{tabular}

Se observa un efecto significativo de las orientaciones motivacionales en el rendimiento académico, $F(2,75)=8,24, p<.001$. Las pruebas post hoc de Bonferroni muestran que la orientación al aprendizaje presenta un rendimiento académico que resulta significativamente superior al de la orientación a la evitación $(p=.000)$ y al de lucimiento $(p=.002)$. El rendimiento académico no difiere significativamente entre la orientación a la evitación y al lucimiento. Los niveles más altos de rendimiento académico son alcanzados por los alumnos orientados al aprendizaje.

Así mismo se aprecia un efecto significativo del género sobre el rendimiento académico, $F$ $(1,75)=5,23, p=.025$. Las mujeres alcanzan niveles de rendimiento académico más alto y se diferencian significativamente de sus pares varones. (Tabla 2) 
Por último, no resulta significativa la interacción entre las orientaciones motivacionales y el género sobre el rendimiento académico, $F(2,75)=1,66, \quad p=.197$.

Los niveles más altos de rendimiento académico son alcanzados por los alumnos motivados por el aprendizaje, independientemente del género. A su vez son las mujeres quienes obtienen mejores niveles de rendimiento académico independientemente de su orientación motivacional.

\section{DISCUSIÓN Y CONCLUSIONES}

El presente estudio buscaba conocer en qué medida el rendimiento académico está influenciado por las orientaciones motivacionales y el género de los estudiantes. Se evaluaron dichas orientaciones en una muestra de estudiantes de bachillerato y se relevaron las calificaciones de los estudiantes como medida de rendimiento académico.

En primer lugar, se ha observado que las distintas orientaciones motivacionales se diferencian significativamente en distintos niveles de rendimiento académico. Particularmente, es la orientación por el aprendizaje la que redunda en niveles más altos de rendimiento. El rendimiento académico de los alumnos orientados al aprendizaje es superior y se diferencia significativamente del de los alumnos orientados a la evitación y al lucimiento.

De acuerdo a la hipótesis planteada se esperaban rendimientos superiores considerando que la orientación al aprendizaje implica estrategias más profundas de procesamiento. Si bien esto suena muy razonable, no siempre se ha observado en los estudios empíricos dado que el rendimiento académico es una variable sobredeterminada (Kaplan y Maehr, 2007; Winne y Nesbit, 2010).

Al interpretar este resultado se debe recordar que la muestra es de estudiantes de bachillerato de nivel socioeconómico medio-alto. Podrían analizarse este sesgo entendiendo que en un contexto exigente, que ofrece condiciones para el aprendizaje y con alumnos que han sorteado previamente las barreras del sistema educativo, la diferencia en las orientaciones motivacionales llega a traducirse en el rendimiento académico.

Aunque lejos de constituir la solución de los problemas de la educación, manipular las variables del contexto de clase para favorecer la motivación por el aprendizaje sigue siendo un camino válido y que puede dar fruto. En este sentido resulta central el rol de los docentes como promotores de orientaciones motivacionales que favorezcan el aprendizaje, mediante la construcción de entornos motivacionalmente ricos.

Por el contrario los alumnos orientados a la evitación y al lucimiento muestran menores niveles de rendimiento académico. A su vez no se encuentran diferencias estadísticamente significativas en el rendimiento académico de estos dos grupos. Cabe recordar que en estudios previos la orientación al lucimiento se ha relacionado tanto con resultados positivos como negativos, en tanto la orientación a la evitación se ha relacionado con niveles más bajos de rendimiento académico (Linnenbrink, 2005; Pintrich y Schunk, 2006).

En el presente estudio los estudiantes orientados a la evitación obtuvieron un rendimiento académico inferior aunque no se diferenciaron significativamente de sus pares orientados al lucimiento. Se podrían considerar ambas orientaciones en el grupo que Boekaerts y Corno (2005) llaman metas de bienestar en el contexto académico, donde las acciones que primar buscan proteger el yo y por tanto los procesos que se activan no son necesariamente los que llevan al mejor aprendizaje. En este modelo las claves contextuales juegan un papel fundamental, en tanto, el sujeto frente a indicios de situaciones amenazantes activan metas de bienestar, tratando de poner a salvo su autoestima. En concordancia con los planteamientos de Dweck y Elliot (1983), estos sujetos más que perseguir aprender evitarán juicios negativos, más que encaminar su acción al aprendizaje, reducirán su esfuerzo, empleando estrategias superficiales que a su vez los conducen a peores rendimientos, como parece mostrar este estudio.

Nuevamente resulta central para este tipo de estudiantes orientados a la ejecución -en su variante de evitación-, que el contexto propicie el empleo de estrategias de procesamiento profundo, y metas de aprendizaje. Por otro lado, es importante que el contexto educativo contribuya a salvaguardar la autoestima de este 
tipo de estudiantes. Claro está que el papel del contexto en el desarrollo de la motivación constituye uno de los tópicos a seguir analizando en la actualidad.

En segundo lugar, se ha observado que las mujeres obtienen niveles de rendimiento académico superiores y se diferencian significativamente de sus pares varones en este aspecto. Para comprender estos resultados seguramente se deba apelar a variables contextuales que están operando de modo diferencial según el género. A su vez, deberán considerarse con más detalle las distintas metas que pueden llevar a varones y mujeres a diferentes resultados.

Seguramente será una línea de trabajo que requerirá de más estudios, a la vez que podría abrir posibilidades a la intervención educativa. La teoría de metas atiende a cómo el contexto de clase moldea el tipo de orientación a metas de los estudiantes, dado que las metas se consideran dependientes del contexto y pasibles de ser modificadas, se han identificado distintas dimensiones de la clase que inciden en la motivación en el contexto de aula: el tipo de tarea, la autoridad, el reconocimiento de los estudiantes, los grupos y la administración del tiempo (Epstein, 1989 en Pintrich y Schunk, 2006). Se podría considerar que algunas de estas dimensiones podrían operar de modo diferencial según el género del estudiante, estudios futuros deberían avanzar en esta dirección.

Así mismo, dada la comprensión teórica actual de los procesos motivacionales y el aprendizaje, será valioso complementar la evaluación de la orientación a metas mediante cuestionarios de auto-informe, con medidas de autorregulación y del clima motivacional del aula.

Si bien en el presente estudio se ha analizado el rendimiento académico como variable dependiente. Sería interesante proponer intervenciones motivacionales que permitan establecer relaciones causales entre las orientaciones motivacionales y el rendimiento académico. Asu vez, futuros estudios deberían profundizar en la evaluación de las orientaciones motivacionales de estudiantes de distintos contextos y en distintos tramos del sistema educativo. Estudios de intervención y longitudinales contribuirán a una mejor comprensión de cómo las variables contextuales configuran el desarrollo desarrollo de las variables motivacionales (Kaplan y Flum, 2010; Urdan y Shoenfelder, 2006).

Avanzar en la descripción de las distintas orientaciones motivacionales de los estudiantes de Educación Media y su relación con el rendimiento académico aún requiere de más estudios. Se espera que dicho conocimiento permita contribuir al diseño de propuestas educativas que contemplen las variables motivacionales. De ese modo favorecer el logro de mayores niveles de desempeño de los estudiantes, no entendidos únicamente como mejores calificaciones sino como fruto de estrategias de autorregulación capaces de propiciar aprendizajes complejos y de calidad.

\section{REFERENCIAS}

Alemán, M. J. (2011). Orientaciones motivacionales y rendimiento académico en estudiantes de bachillerato. Memoria de grado Inédita: Universidad Católica del Uruguay.

Alonso Tapia, J. (2001). Motivación y estrategias de aprendizaje. Principios para su mejora en alumnos universitarios. En García, A.; Muñoz-Repiso,V. (coord.) Didáctica Universitaria. Madrid: La Muralla

Alonso Tapia, J. (2005). Motivaciones, expectativas y valores-intereses relacionados con el aprendizaje: El cuestionario MEVA. Psicothema, 17 (3), 404-411.

Boekaerts, M., \& Corno, L. (2005). Self-regulation in the classroom: a perspective on assessment and intervention. Applied Psychology: An International Review, 54 (2), 199-231.

Cerezco, M. \& Casanova, P. (2004). Gender differences in academic motivation of secondary school student. Electronic Journal of Research in Educational Psychology, 2 (1), 97-112.

Delgado, B., Inglés, C., García-Fernández, J., Castejón, J., \& Valle, A. (2010) Diferencias de género y curso en metas académicas en alumnos de Educación Secundaria Obligatoria. Revista española de pedagogía, 245, 67-84.

Dweck, C. \& Leggett, E. (1988). A social-cognitive approach to motivation and personality. Psychological Review, 95, 256-273.

Dweck, C., \& Elliot, E. (1983). Achievement motivation. En Mussen, P. \& Heatherington, M. Handbook of child psychology, 4, Socialization, personality and social development. New York: Wiley.

Efklides, A. (2011). Interactions of metacognition with motivation and affect in self-regulated learning: The MASRL Model. Educactional Psychologist, 46(1), 6-25. 
Elliott, E., \& Dweck, C. (1988). Goals: An Approach to Motivation and Achievement, Journal of Personality and Social Psychology, 54 (1), 5-12.

Elliot, E. \& Harackiewicz, J. (1996). Aproach and Avoidance achievement goals and intrinsic motivation: A meditational Analysis, Journal of Personality and Social Psychology, 70, 461-475.

Elliot, E. \& Church, M. A. (1997). A hierarchical model of approach and avoidance achievement motivation, Journal of Personality and Social Psychology, 72, 218-232.

Harackiewicz, J., Barron, K. \& Elliot, E. (1998). Rethinking achievement goals: When are they adaptative for college student and why? Educational Psychologist, 33, 1-21.

Harackiewicz, J., Barron, K., Tauer, J. M., Carter, S., \& Elliot, A. (2000). Shortterm and long-term consequences of achievement goals: Predicting interest and performance over time. Journal of Educational Psychology, 92, 316-330.

Harackiewicz, J.; Barron, K..; Pintrich, P.; Elliot, A. \&Thrash, T. (2002). Revision of achievement goal theory: Necessary and illuminating. Journal of Educational Psychology, 94 (3), 638-645.

Huertas, J.A. (2008). Las teorías de la motivación desde el ámbito de lo cognitivo y lo social. En Palmero, F. \& Martínez, F. Motivación y Emoción. Madrid: McGraw-Hill.

Kaplan, A., \& Flum, H. (2010). Achievement goal orientations and identity formation styles. Educational Research Review, 5(1), 50-67.

Kaplan, A., \& Maehr, M. L. (2007). The Contributions and Prospects of Goal Orientation Theory. Educational Psychology Review, 19(2), 141-184.

Linnenbrink, E. (2005). The dilemma of performanceapproach goals: the use of multiple goal contexts to promote students' motivation and learning. Journal of Educational Psychology, 97 (2), 197-213.

Meece, J.; Blumenfeld, P.; \& Hoyle, R. (1988). Student's goal orientation and cognitive engagement in classroom activities. Journal of Educational Psychology, 80, 514-523.

Meece, J.; Bower, B. \& Burg, S. (2006). Gender and Motivation. Journal of School Psychology, 44, 351-373.
Meece, J. \& Miller, S. (1990). A longitudinal analysis of elementary school student's achievement goals in literacy activities. Contemporary Educational Psychology, 26, 454-480.

Midgley, C., Kaplan, A., \& Middleton, M. (2001). Performance-approach goals: Good for what, for whom, under what circumstances, and at what cost? Journal of Educational Psychology, 93, 77-86.

Pintrich, P. (2003). A Motivational Science Perspective on the Role of Student Motivation in Learning and Teaching Contexts. Journal of Educational Psychology, 95(4), 667-686.

Pintrich, P.; \& Schunk, D. (2006). Motivación en contextos educativos. Madrid: Pearson.

Risso, A., Peralbo, M., \& Barca, A. (2010). Cambios en las variables predictoras del rendimiento escolar en Enseñanza Secundaria. Psicothema, 22, 790-796.

Rodríguez, M. N. (2010). Perfiles motivacionales definidos mediante análisis de conglomerados y su relación con la capacidad percibida y el rendimiento académico. Anales de Psicología, 26 (2), 348-358.

Rodríguez Moneo, M. \& Huertas, J.A. (2000). Motivación y cambio conceptual. Tarbiya, Revista de Investigación e Innovación Educativa, 26, 51-71.

Urdan, T., \& Schoenfelder, E. (2006). Classroom effects on student motivation: Goal structures, social relationships, and competence beliefs. Journal of School Psychology, 44 (5), 331-349.

Valle, A; González Cabanach, R; Nuñez, J.C; GonzálezPienda, J.A. Rodriguez Martínez, S; Rosário, P. (2009) Perfiles Motivacionales en estudiantes de secundaria: Análisis diferencial en estrategias cognitivas, estrategias de autorregulación y rendimiento académico. Revista Mexicana de Psicología, 26 (1), 113-124.

Winne, P. H., \& Nesbit, J. C. (2010). The psychology of academic achievement. Annual review of psychology, 61, 653-78.

Yeung, A.; Lau, S. \& Nie, Y. (2011). Primary and secondary students' motivation in learning English: Grade and gender differences. Contemporary Educational Psychology, 36, 246-256.

Para citar este artículo:

Alemán, M.J, Trías, D. \& Curione, K. (2011). Orientaciones motivacionales, rendimiento académico y género en estudiantes de bachillerato. Ciencias Psicológicas V (2): 159-166. 